\title{
Damping and drive of low frequency modes in tokamak plasmas
}

\author{
Ph. Lauber, S. Günter \\ Max-Planck-Institut für Plasmaphysik, EURATOM-Association, Garching, Germany \\ E-mail: Philipp.Lauber@ipp.mpg.de
}

\begin{abstract}
.
The ability to predict the stability of fast-particle-driven Alfvén eigenmodes in burning fusion plasmas requires a detailed understanding of the dissipative mechanisms that damp these modes. In order to address this question, the linear gyro-kinetic, electromagnetic code LIGKA [11], [12] is employed to investigate their behaviour in realistic tokamak geometry.

Recently, the model and the implementation of LIGKA were extended in order to capture rigorously the coupling of the shear Alfvén wave to the drift and sound waves. This coupling becomes important for the investigation of low frequency modes like the Alfvén cascade modes (AC), the beta-induced Alfvén eigenmodes (BAE), the geodesic acoustic modes (GAM), the energetic particle modes (EPM) and - at even lower frequencies - the resistive wall modes (RWM).

The authors report on an effort to close the gap between high-n micro-scale turbulence codes (in their linear phase) and low-n global MHD codes. More precisely, the aim is to describe both regimes within the same framework of equations and with the same numerical implementation by improving the range of applicability and validity of LIGKA: an eigenvalue code allows to explore both the local complex dispersion relations for e.g. kinetic Alfvén waves (electromagnetic), ion acoustic waves (electrostatic) or drift waves in realistic geometry and also the their global eigenfunctions.

As an application of this extension, an investigation of the kinetic RWM damping mechanisms is carried out.
\end{abstract}

\section{Introduction}

The destabilising effect of fusion-born $\alpha$-particles on global MHD modes is of great interest because of their serious consequences for plasma heating and plasmawall interaction. In order to make predictions for the stability of such modes in a predominantly $\alpha$-particle heated plasma like ITER, the background damping mechanisms of these modes have to be investigated carefully. These mechanisms are electron and ion Landau damping, continuum damping, collisional damping and radiative damping. The latter mechanism requires a non-perturbative description, since the MHD properties of the mode structures are modified by coupling to the kinetic waves e.g. the kinetic Alfvén wave (KAW) [2]. Examples for these modes are the 
toroidal Alfvén eigenmodes (TAEs) [3],[4]that exist in gaps in the Alfvén continuum due to toroidal coupling, or the Alfvén cascade modes (ACs) [5] that can exist if the q-profile exhibits a local minimum or is very flat. Extensive theoretical and numerical investigations have been carried out concerning their drive [1][6] and damping $[7][8][9][10]$.

There are two reasons why the accurate determination of damping rates is particularly difficult: firstly, the damping rates for modes of the very same type can vary over several orders of magnitude depending on the different plasma background conditions, i.e. the mode can be exponentially sensitive to these conditions. Secondly, the spatial structure of the mode can range from sub-ion-radius scales up to the dimensions of the whole plasma, i.e. from millimetre to metre for typical tokamak parameters. Therefore, a tool is needed that describes both micro and macro scales within the same model and the same numerical implementation.

In order to address this problem the linear eigenvalue code LigkA [11],[12] has been extended in several aspects. LIGKA was originally designed for global modes in the TAE frequency regime (TAEs, kinetic TAEs, Alfvén Cascades). It is based on a selfconsistent model by Qin et al. [14][15] consisting of the quasi-neutrality equation and the moment equation for the perturbed current. It is non-perturbative, since it allows for a non-linear dependence of the eigenvalue through the velocity space integrals and also for a mode structure which is not prescribed by ideal MHD calculations. The propagator integrals along the drift orbit of ions and electrons are carried out numerically using the drift kinetic code HAGIS [16],[17], and the resonance integrals are solved accurately applying rational interpolation and grid refinement techniques.

However, as shown in section 2, the model also describes small scale and lowfrequency phenomena such as ion acoustic, electrostatic and electromagnetic drift waves. Therefore, no changes in the equations themselves had to be made, i.e. the theoretical model of the 'old' and the 'new' LIGKA remained the same. On the numerical side, a few improvements and extensions were necessary to solve this set of equations in the low-frequency limit: firstly, because of $\omega_{\text {mode }} \sim \omega_{d} \sim \omega_{b, t}$, two poles in the resonance denominator for the background species (see eqns 7,8) appear frequently instead of usually one pole for high frequency modes. Therefore, the rational interpolation scheme for the denominator [12] had to be adopted. Secondly, the grid refinement in the radial direction was improved to check if the assumption $k_{\perp} \varrho_{i} \leq 1$ is fulfilled for the 'new' small-scale-modes . (LigkA employs an expansion of the Bessel function up to second order, that is relatively accurate for $k_{\perp} \varrho_{i} \leq 0.5$ and still acceptable for $0.5 \leq k_{\perp} \varrho_{i} \leq 1$ ). Thirdly, the matrix build-up and storage procedure was improved to account for the coupling of up to 20 poloidal modes. (The old LIGKA version allowed for maximal 9). Finally, a local solver allows to trace the real and imaginary part of the dispersion relation throughout the whole plasma, making comprehensive comparisons of global and local results possible. Basic tests and benchmarks are shown in section 3.

As a first application of this extension, the kinetic damping of the resistive wall mode (RWM) is investigated. If there is no conducting vessel wall close to the plasma edge, 
this mode is essentially a rather global, pressure driven external kink mode that grows very fast i.e. on the typical MHD timescale. In the presence of a wall, the growth rate of the mode is slowed down to the resistive time scale of the wall due to the generation of mirror currents in the wall that counteract the mode perturbation. This slowly growing mode can be stabilised by toroidal plasma rotation or a set of external feedback coils [18], [19]. In order to predict the critical rotation frequency that is needed to stabilise the mode, the kinetic damping mechanisms have to be studied carefully. So far simple, perturbative MHD models based on an extended energy principle have been used [20]. Magnetic and diamagnetic drifts as well as electrostatic coupling were neglected there. LIGKA includes this missing physics. However, so far there is no vacuum part implemented in LIGKA. Therefore, the ideal MHD code CASTOR [21] is applied to calculate the vector potential of the ideal MHD eigenfunction that is used as boundary condition on the last closed flux surface for the vector potential in LIGKA. Details and results are presented in section 4 .

\section{Model, relevant limits and numerics}

LigkA solves the following system of equations [14],[15],[11][12]: the linearised gyrokinetic equation, where $h$ is the non-adiabatic part of the perturbed distribution function $f$ :

$$
\begin{aligned}
& h_{a}=-i e_{a} \frac{e^{i(n \varphi-\omega t)}}{\sum_{m}} \int_{-\infty}^{t} d t^{\prime} e^{i\left[n\left(\varphi^{\prime}-\varphi\right)-m\left(\theta^{\prime}-\theta\right)-\omega\left(t^{\prime}-t\right)\right]} e^{-i m \theta} \\
& \cdot \frac{\partial F_{0 a}}{\partial E}\left(\omega-\omega_{* a}\right) J_{0} \cdot\left[\phi_{m}\left(r^{\prime}\right)-\left(1-\frac{\omega_{d a}}{\omega}\right) \psi_{m}\left(r^{\prime}\right)\right]
\end{aligned}
$$

the quasi-neutrality equation:

$$
\sum_{a} \frac{e_{a}^{2} n_{a}}{T_{a}}\left[\varrho_{a}^{2} \nabla_{\perp}^{2}\right] \phi+e_{a} \int J_{0} f_{a} d^{3} \mathbf{v}=0 ; \quad \mathbf{E}=-\nabla \phi-\frac{\partial \mathbf{A}}{\partial t} ; \quad A_{\|}=\frac{1}{i \omega}(\nabla \psi)_{\|}
$$

and the gyrokinetic moment equation:

$$
\begin{aligned}
& -\frac{\partial}{\partial t}\left[\nabla \cdot\left(\frac{1}{v_{A}^{2}} \nabla_{\perp} \phi\right)\right]+(\mathbf{B} \cdot \nabla) \frac{\nabla \times \nabla \times \frac{c}{i \omega}(\nabla \psi)_{\|}}{B^{2}}+\left[\frac{1}{i \omega} \nabla(\nabla \psi)_{\|} \times \mathbf{b}\right] \cdot \nabla \frac{\mu_{0} j_{0 \|}}{B} \\
& =-\sum_{a} \mu_{0} \int d^{3} v\left(e \mathbf{v}_{d} \cdot \nabla J_{0} f\right)_{a}+\frac{3}{4} \frac{\mu_{0} e_{a}^{2} n_{a}}{T_{a}} \varrho_{a}^{4} \nabla_{\perp}^{4} \frac{\partial}{\partial t} \phi
\end{aligned}
$$

Here, $\sum_{a}$ indicates the sum over different particle species with the perturbed distribution function $f_{a}$, mass $m_{a}$, charge $e_{a}$, unperturbed density $n_{a 0}$, thermal velocity $v_{t h, a}=$ $\sqrt{T_{a} / m_{a}}$, cyclotron frequency $\Omega_{a}$ and gyro-radius $\varrho_{a} . \phi$ is the perturbed electrostatic potential and $\psi$ is a measure for the electromagnetic potential according to equation 2 , $\omega_{* a}=\frac{\mathbf{b} \times \nabla F_{0 a}}{i e_{a} B} / \frac{\partial F_{0 a}}{\partial E} \cdot \nabla$ is the diamagnetic frequency and $\omega_{d a}=-i \mathbf{v}_{d a} \cdot \nabla$ is the magnetic drift frequency acting on the perturbed potentials. $j_{0 \|}$ is the equilibrium background current and $v_{A}$ the local Alfvén speed.

Simple limits of these equations can be obtained, e.g. the 'reduced kinetic limit' [22], [23]:

$$
\left[1+\xi_{e} Z\left(\xi_{e}\right)+1+\xi_{i} Z\left(\xi_{i}\right)\right](\phi-\psi)=T_{e} / T_{i} \varrho_{i}^{2} \nabla_{\perp}^{2} \phi
$$




$$
\nabla_{\perp} \cdot \frac{\omega^{2}}{v_{A}^{2}} \nabla_{\perp} \phi+\frac{\partial}{\partial s} \nabla_{\perp}^{2} \frac{\partial \psi}{\partial s} \quad=\frac{3}{4} \varrho_{i}^{2} \frac{\omega^{2}}{v_{A}^{2}} \nabla_{\perp}^{4} \phi
$$

Applying the hot electron, cold ion expansion immediately gives the Alfvén branch, the KAW, the ion acoustic wave and the drift wave:

$$
\varrho_{s}^{2} k_{\perp}^{2} \phi=\left(\frac{c_{s}^{2} k_{\|}^{2}}{\omega^{2}}-1+\frac{\omega_{* e}}{\omega}\right)(\phi-\psi) ; \quad \frac{\omega^{2}}{v_{A}^{2}} \phi-k_{\|}^{2} \psi=0
$$

The basic version of LigKA solves equations (2), (3) and the linear gyro-kinetic equation (1) for the perturbed distribution function $f$ up to 2 nd order in $k_{\perp} \varrho_{i}$. Straight field line coordinates for the background quantities given by the equilibrium code HELENA [24] are chosen. LigkA has been extended to calculate correctly the residual part of the Landautype integrals for the case of negative growth rates, i.e. damped modes. It uses a rational interpolation scheme for the resonance denominator (see formula 8 )which allows for accurate and fast evaluation of the pole contributions without employing derivatives. In particular for the low frequency extension, multiple poles in the resonance denominator (due to $\omega_{b, t}$ and $\omega_{d}$ ) have to be accounted for. Furthermore, a very fine radial grid is needed to resolve the small scale modes near the rational surfaces. Grid refinement techniques are also applied for the velocity space integration. When examining the rich spectrum over a large frequency domain, sometimes many closely spaced modes are expected. Using a Nyquist solver is cumbersome under these conditions because a large number of poles require a high number of sample points along the integration contour. Thus an antenna-like version of LIGKA was developed: A drive vector is added to the right hand side of the homogeneous equation:

$$
M(\omega)\left(\begin{array}{c}
\phi \\
\psi
\end{array}\right)=\mathbf{d}
$$

where $\mathbf{d}$ is only nonzero for the last finite element at the plasma edge, prescribing a small perturbation from the outside. The eigenfunctions are found by inverting $M(\omega)$ resulting in:

$$
\mathbf{I}\left(\begin{array}{c}
\phi \\
\psi
\end{array}\right)=M(\omega)^{-1} \mathbf{d}
$$

and the plasma response is 'measured' by an integral over the eigenfunction:

$$
\mathcal{R}=\sum_{m} \int_{0}^{a} \phi_{m} \phi_{m}^{*} d r
$$

\section{Low Frequency Benchmarks}

For a model equilibrium based on JET parameters, $B_{0}=3.53 \mathrm{~T}, R_{0}=2.96 \mathrm{~m}, \beta \approx 2 \%$, a flat density profile $(\nabla n / n=0.01)$ and the other relevant profiles given in figure 1 , the local continua are determined by a local solve of the complex matrix coefficients. For comparison with analytical theory at this point just one harmonic is kept : $m=1$, $n=1$. Employing a Nyquist contour integration for determining the residue enclosed by a circle in the complex plane, allows to find up to 20 eigenvalues at the same time (see 

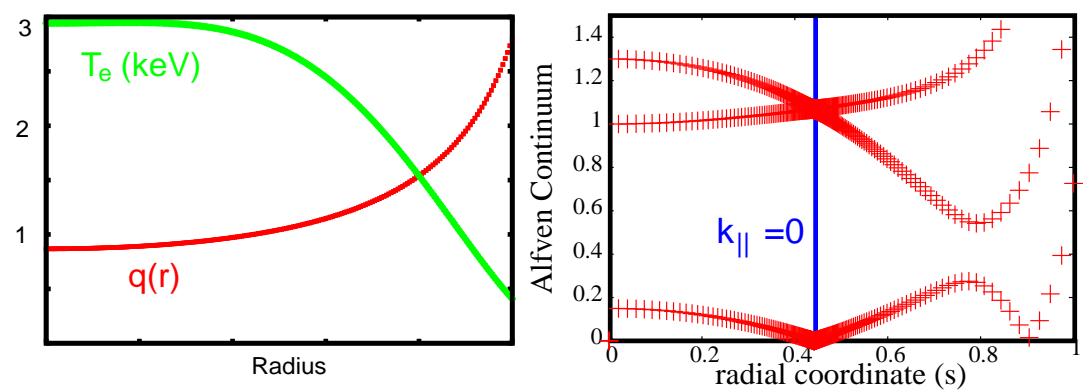

Figure 1. $q$, temperature profile and the corresponding Alfvén continuum
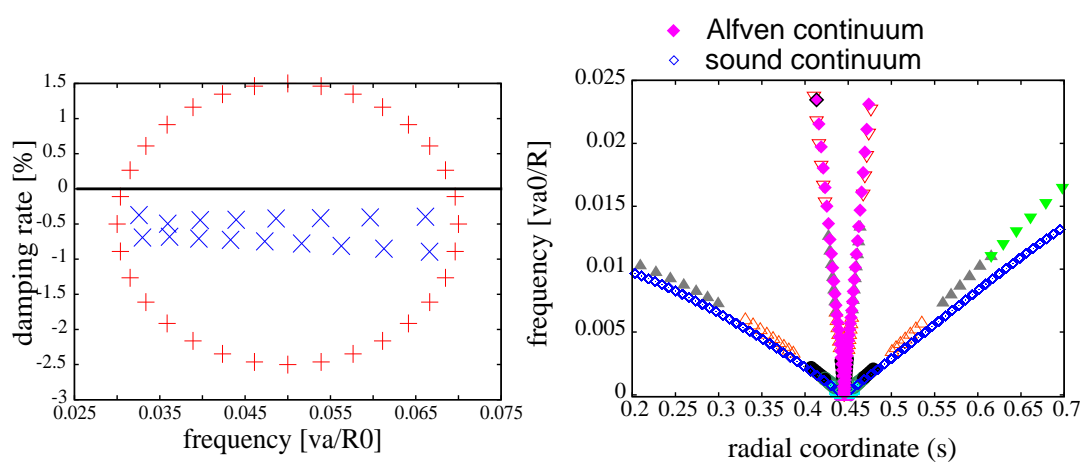

Figure 2. Nyquist contour including roots in the complex plane and the real part of the KAW/IAW dispersion relation. The MHD solutions are over-plotted as described in the right plot. Different colours/symbols refer to different integration circles.

figure 2). However, for very small eigenvalues which require high accuracy the circles have to be smaller including less roots. Typically 16 to 32 points on the contour are enough for this local solve.

Clearly, two branches, the KAW branch and the ion acoustic (IAW) branch can be identified by comparison with the formulae $\omega=k_{\|} v_{a}$ and $\omega=k_{\|} c_{s}$. Figure 3 shows the real (left) and the imaginary (right) part of the dispersion relation in logarithmic scale. For this local solve the quantity $k_{\perp} \varrho_{i}$ has been set to 0.1 and $T_{e}=T_{i}$. The radial coordinate close to the $q=1$ surface can also be seen as $k_{\|}$coordinate. Both branches are stable, the IAW is heavily damped. One can compare the KAW damping rate with the simple slab result

$$
\gamma / \omega \approx-\frac{1}{2} \sqrt{\frac{\pi}{2}} \sqrt{\frac{m_{e}}{m_{i}}} \frac{k_{\perp}^{2} v_{A}}{\Omega_{i}^{2}} c_{s} \approx 0.38 \% \quad\left(k_{\perp} \varrho_{i}=0.1\right)
$$

and finds that this expression gives a lower limit for the damping rate except very close to the $k_{\|}=0$ surface. For $k_{\|}=0$ the real part of the dispersion relation reduces to $\omega=\omega_{* e}$, the electrostatic drift wave, which was chosen to be very small via a flat density gradient as a test for the numerics.

One can make this sound branch more unstable by setting $T_{e}>T_{i}$ and increasing the density gradient. The resulting dispersion relation is shown in figure 4 for the parameters $T_{e}=3 T_{i}$ and $\nabla n / n=0.9$. 

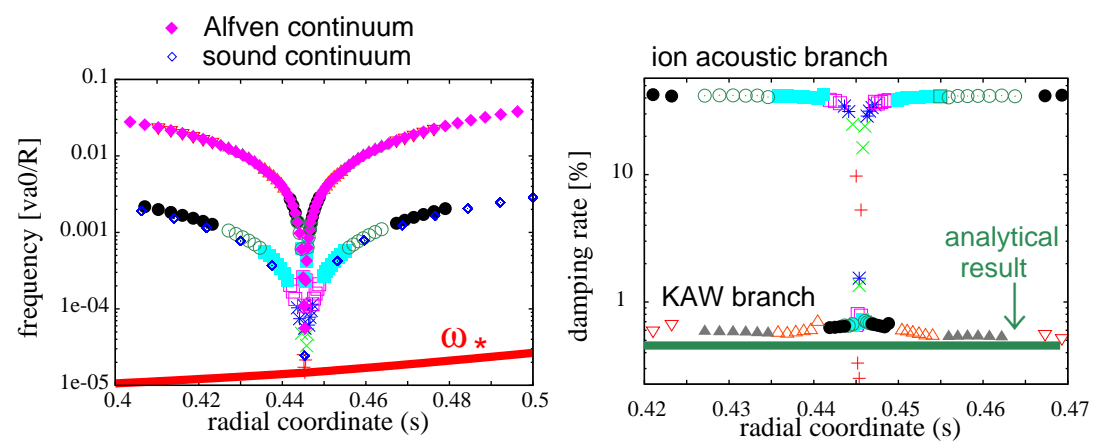

Figure 3. Real and imaginary part of the dispersion relation for the KAW and IAW. The different colours again refer to different contours in the complex plane that are identical to the contours in figure 2
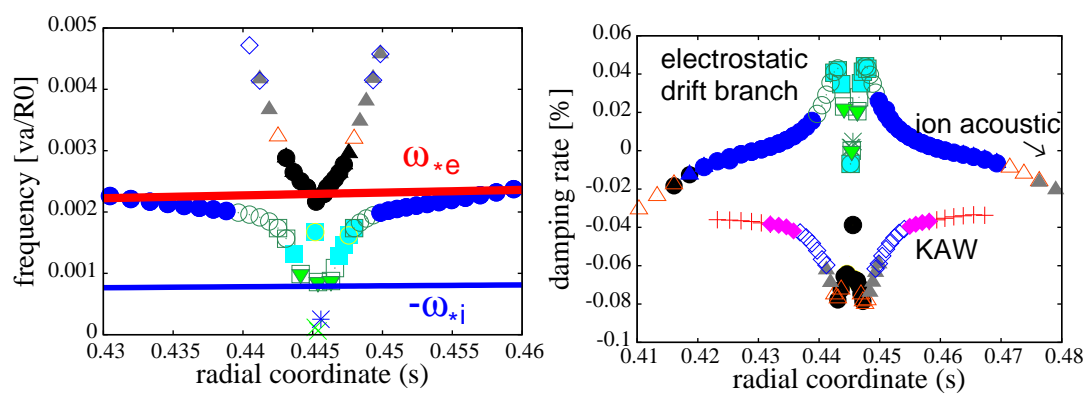

Figure 4. Real and imaginary part of the dispersion relation for the KAW and IAW for $T_{e}=3 T_{i}$ and $\nabla n / n=0.9$. Different colours refer to different contours in the complex plane.

The two branches couple now stronger to the drift wave: the ion acoustic branch turns into the electrostatic drift branch, the KAW turns into the drift Alfvén wave. Again the two limits of $\omega=\omega_{* e}$ resp. $\omega=-\omega_{* i}$ are found in the real part of the spectrum. The imaginary part shows a small unstable region for the electrostatic drift wave under these conditions.

After these checks for the correct implementation and numerical accuracy, one can start to solve the system globally. This requires considerably more time: it is much faster to solve 256 (number of radial grid points) times a $4 \times 4$ matrix (for one poloidal harmonic) than to solve once a $(2 * 256) \times(2 * 256)$ matrix. Additionally, it is much harder to obtain convergence, i.e. finding the zeros of the determinant of a large matrix. However, the combination of the Nyquist contour solver and a 2d Newton method allows one to find converged solutions in typically about 32 (Nyquist) +10 (Newton) evaluation steps. Figures 5 and 6 show two global solutions for the KAW wave and the IAW. The red and the blue line refer to the electrostatic and the electromagnetic potential, respectively. The crosses indicate the actual grid points (of the electromagnetic potential) of the calculation. Clearly, the KAW is well resolved (almost 10 grid points per wavelength) in the critical region. When comparing the KAW in figure 5 and the IAW in figure 6 it becomes obvious that the KAW is an electromagnetic mode where both potentials 

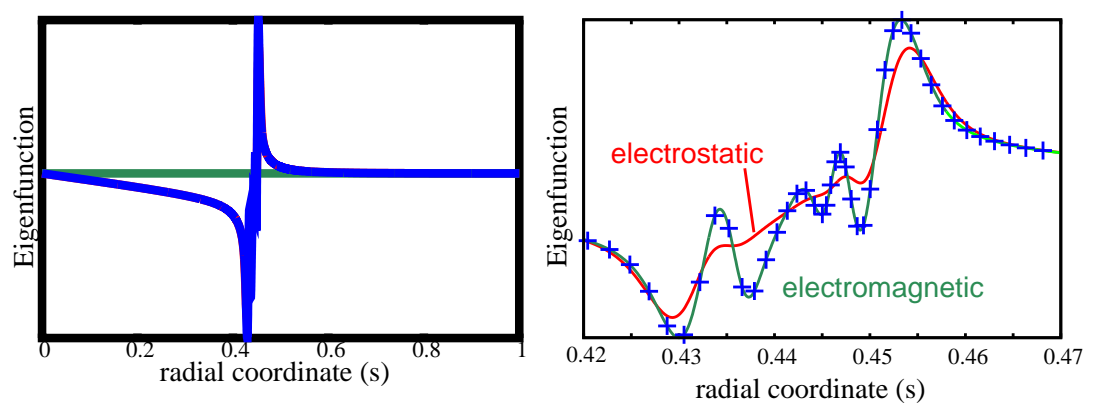

Figure 5. Global eigenfunction of a KAW, details on the right. Electrostatic and electromagnetic potentials are shown. The crosses on top of the electromagnetic potential indicate the actual grid points used in the simulation. The maximal $k_{\perp} \varrho_{i}$ is about 0.4 for this mode.
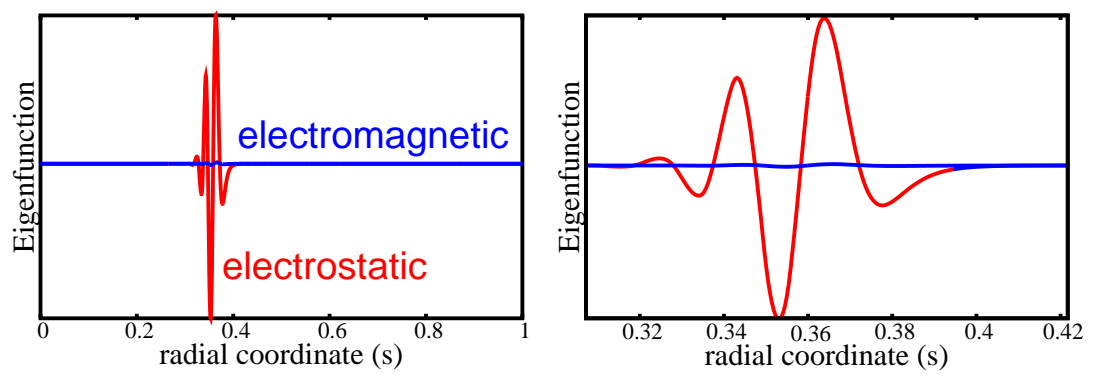

Figure 6. Global eigenfunction of a IAW, the electrostatic part is much larger than the electromagnetic part.

are comparable, in contrast to the IAW where the electrostatic component dominates. As a last question in this section the damping rates of the global modes close to the diamagnetic frequency are investigated. For these calculations, the density gradient was artificially increased such that $\omega_{*}=0.01 \omega_{A}$. As can be seen in figure 7 , the damping rates out of this global calculation are considerably higher than the local ones. The least damped modes are found just below $\omega_{*}$ with a radial mode structure that is again dominated by the electrostatic potential with only a small electromagnetic contribution. In order to destabilise this mode one would need steeper gradients (density and/or temperature), or one would have to go to higher toroidal mode numbers. The instability threshold behaviour (ITG threshold) or also the trapped electron mode is beyond the scope of this paper and will be investigated elsewhere.

\section{Application: Kinetic damping of the resistive wall mode (RWM)}

As shown in the last section, LIGKA is now able to treat small scale modes near rational surfaces with the same system of equations and with the same numerical methods as for the global modes. As an application of this development, we investigate here the kinetic damping mechanisms of the RWM.

Since there is neither a vacuum part nor a model for rotation implemented in LIGKA 


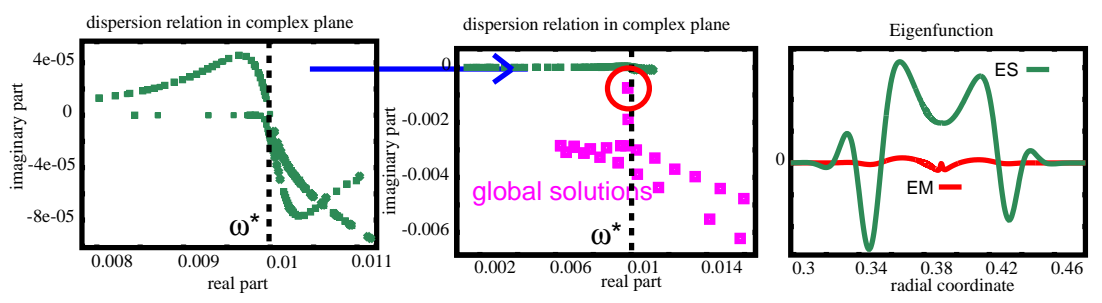

Figure 7. Local damping rates (left) and global damping of the electrostatic drift wave close to the diamagnetic frequency; the eigenfunction on the right corresponds to the encircled (least damped) eigenvalue (in the middle)

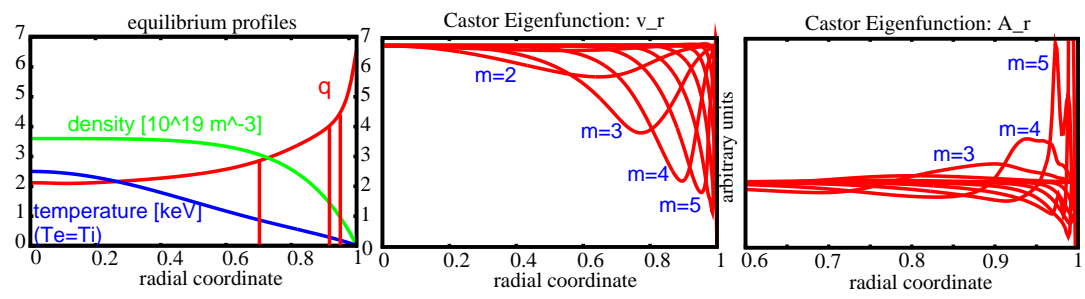

Figure 8. Equilibrium quantities, the radial velocity perturbation $v_{r}$ and the electromagnetic potential perturbation $A_{r}$ for an external kink mode as given by the CASTOR code; on the left also the rational surfaces $q=3$ at $\varrho_{\text {pol }}=0.74, q=4$ at $\varrho_{p o l}=0.92$ and $q=5$ at $\varrho_{\text {pol }}=0.96$ are shown

the problem cannot be treated in a complete self-consistent way. Instead, the ideal MHD-Code CASTOR [21] is used to obtain the eigenfunctions of the external kink mode (see figure 8 for equilibrium quantities, the radial velocity perturbation $v_{r}$ and the electromagnetic potential perturbation $A_{r}, T_{i}=T_{e}$ ). The amplitudes of the poloidal harmonics at the last closed flux surface as given by CASTOR are used as boundary conditions for LIGKA, i.e. as the drive vector (equation 4) of the antenna version. The rotation is therefore modeled by rotating boundary conditions.

Now we can vary the drive frequency and measure the plasma response. It should be noted that for a start the growth rate $\gamma$ of the RWM is assumed to be very small and is therefore set to 0 . A non-zero $\gamma$ will be considered at the end of this section.

Figure 9 shows the response function over a large range of frequency. Of course, frequencies above $\sim 20 \mathrm{kHz}$ cannot be interpreted as a plasma rotation but rather as perturbations generated by external antennas (like e.g. the saddle coil antennas at JET [25]). The maxima correspond to modes that are less damped than the surrounding solutions. Going from high to low frequencies one can easily identify the TAE mode $\left(\omega \approx 0.18 \omega_{A}\right)$, a kinetically modified TAE $\left(\omega \approx 0.165 \omega_{A}\right)$ and the end of a continuumfree region at the edge at about $\left(\omega \approx 0.1 \omega_{A}\right)$. Below that region the damping increases considerably, not only due to the coupling to the KAW but also due to sound wave damping. It is interesting to note, that for weakly damped modes like the TAE mode the details of the excitation i.e. the prescribed external kink perturbation via the drive vector $d$ do not influence the eigenfrequency at all and alter the eigenfunction only slightly very close to the edge. 

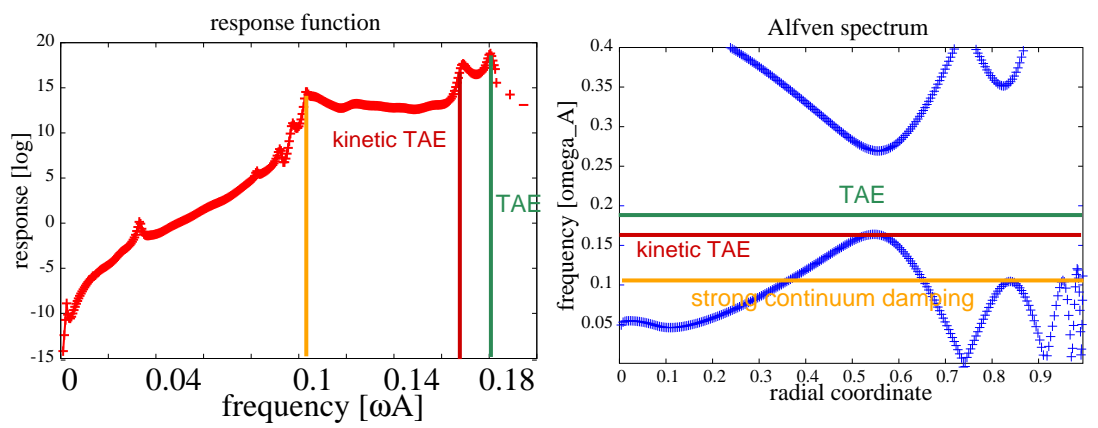

for typical AUG parameters:

$1 \mathrm{kHz} 20 \mathrm{kHz} \quad 70 \mathrm{KHz} \quad 120 \mathrm{kHz}$

Figure 9. Response function (left) and Alfvén continuum (right); details in the text
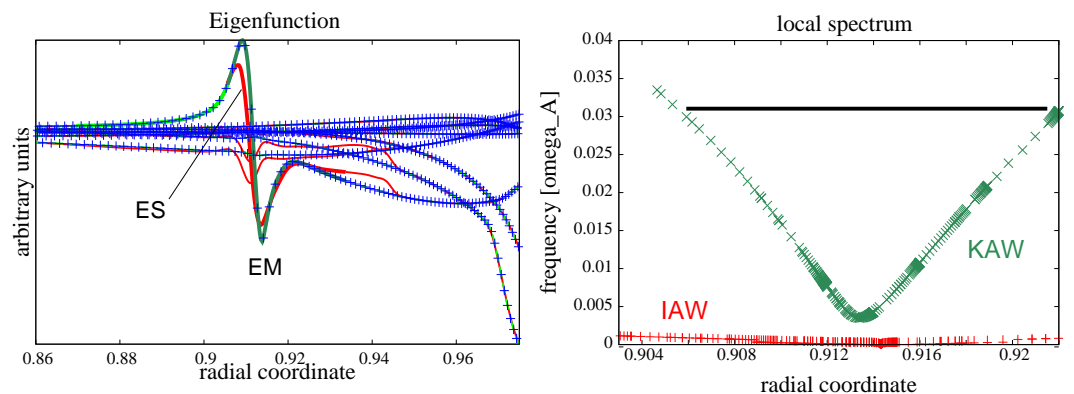

Figure 10. left: electrostatic and electromagnetic eigenfunctions of a micro-tearing mode for $\omega \approx 0.03 \omega_{A}$; right: position of this mode with respect to the local spectrum

The next peak in the spectrum appears at about $\omega \approx 0.03 \omega_{A}$. The corresponding eigenfunction is (figure 10) electromagnetic. From it's shape one can identify this mode as a stable micro-tearing mode close to the $q=4$ rational surface. Figure 10 also shows the position of this mode relative to the local spectrum: it still lies within the KAW branch and therefore is a low-frequency Alfvén-type mode.

This (almost) pure Alfvén-type character is lost when one goes to even lower frequencies. As shown in the last section, at $\omega \leq \omega_{*}$ the KAW and the drift branch couple. Figure 11 shows the response function for these very low frequencies that are now comparable to typical plasma rotation frequencies. For typical ASDEX-Upgrade plasma parameters $0.0025 \omega_{A}$ corresponds to $1.5 \mathrm{kHz}$. The three maxima in the response are due to modes with predominantly electrostatic character localised in a not too narrow region around the $q=3$ and mainly $q=4$ surface. The modes exhibit three resp two resp one maximum of the electrostatic potential near the $q=4$ surface. Therefore the two upper modes can be identified as radial harmonics of the lowest mode. The 3-maxima mode (mode1) is least damped since it is closest to the end of the local KAW branch and therefore finds a rather wide radial region without continuum intersection (see figure 12). Close to the edge the eigenmodes show the typical radial shape of an external kink mode.

In order to identify the main damping mechanism one can use an intermediate 


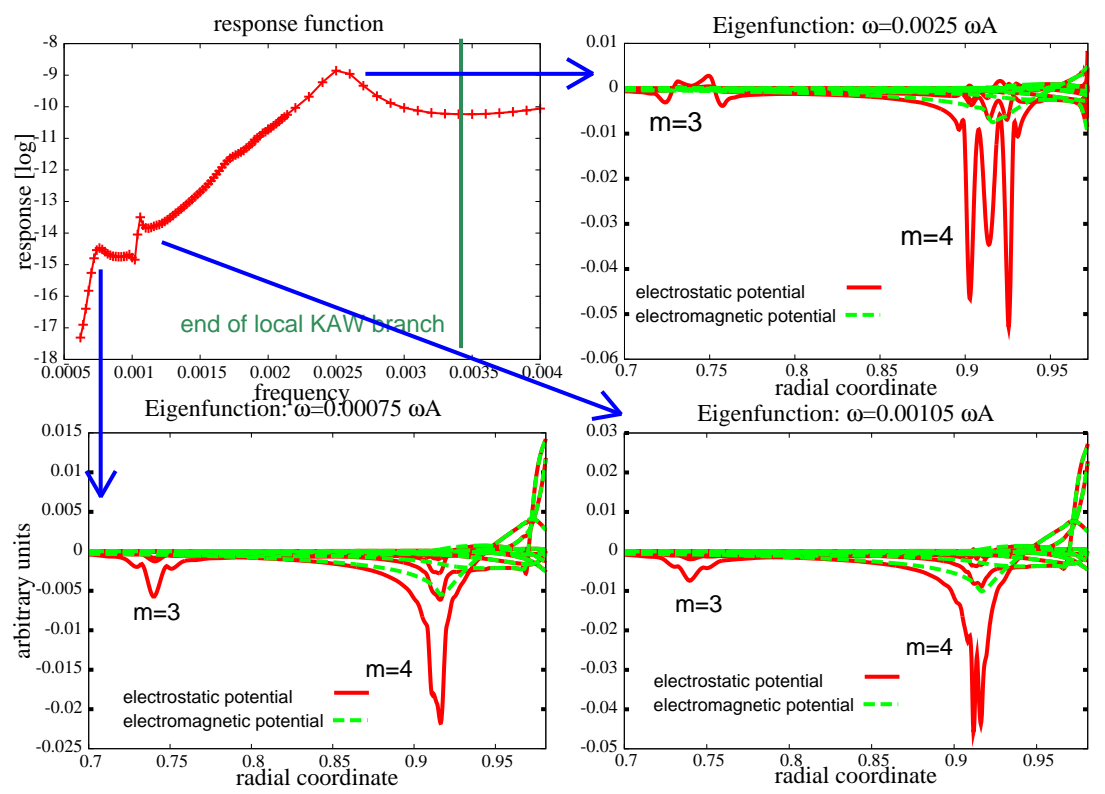

Figure 11. Response function for very low frequencies $\left(\omega \sim \omega_{\text {rot }}\right)$ and the corresponding eigenfunctions

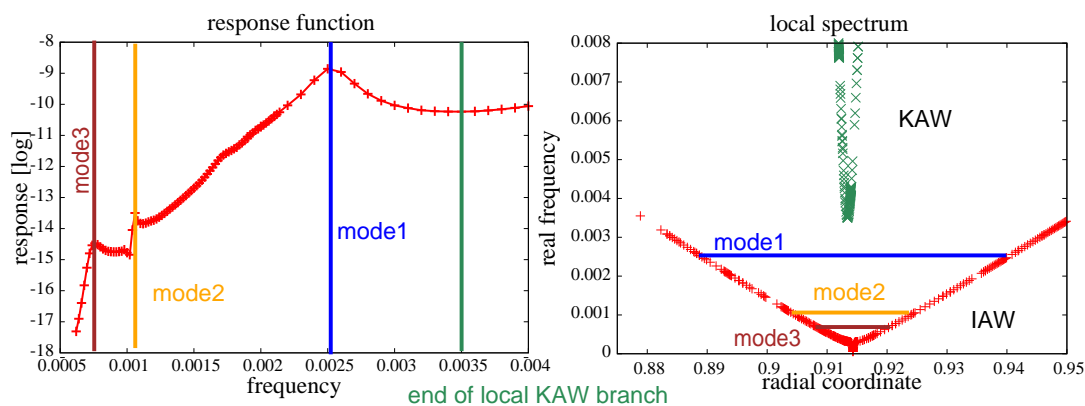

Figure 12. Response function for very low frequencies $\left(\omega \sim \omega_{\text {rot }}\right)$ and the corresponding local spectrum

diagnostic of LIGKA: the imaginary part of the non-adiabatic response for trapped

$$
\mathcal{I M} \int_{\text {trapped }} d v^{3} \sum_{k} \frac{\partial F_{0}}{\partial E} \frac{\left(\omega-\hat{\omega}_{*}\right)}{\omega-\omega_{d}^{0}-k \omega_{b}} K_{m, m^{\prime}, k} \hat{a}_{k m}
$$

and for passing particles:

$$
\mathcal{I} \mathcal{M} \int_{\text {passing }} d v^{3} \sum_{k} \frac{\partial F_{0}}{\partial E} \frac{\omega\left(\omega-\hat{\omega}_{*}\right)}{\omega^{2}-\left(\omega_{d}^{0}-(n q-m+k) \omega_{t}\right)^{2}} K_{m, m^{\prime}, k} \hat{a}_{k m} .
$$

Here $\omega_{t, b}$ are transit resp bounce frequency, $\omega_{d}^{0}$ is the orbit-averaged drift frequency and $K_{m, m^{\prime}, k}$ and $\hat{a}_{k m}$ are orbit-averaged phase factors [14, 12]. Figure 13 shows this quantity for both passing/trapped ions and electrons as a function of the normalised radius. One can see that the damping is mainly due to ions close to the rational surfaces. Trapped ions only play a minor role, the electron contribution completely vanishes. Therefore one can conclude that ion acoustic wave damping is the main kinetic damping mechanism for RWMs. In order to determine if the $q=3$ or the $q=4$ surface contributes more to 


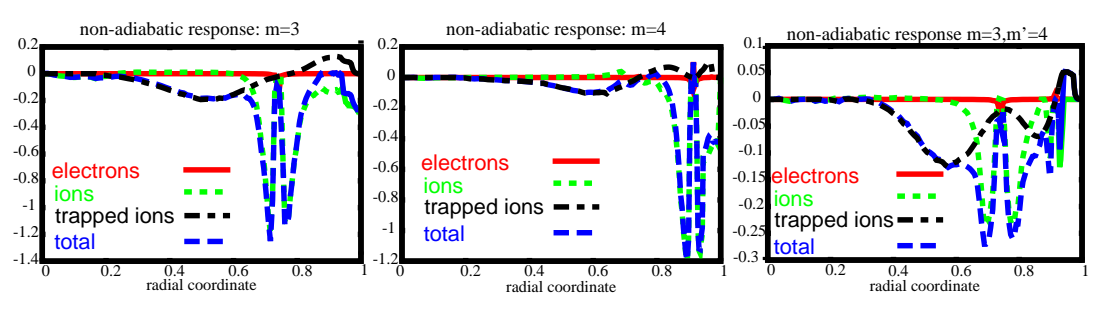

Figure 13. imaginary part of the non-adiabatic kinetic response for $m=3, m=4$ and the sideband contribution $m=3, m^{\prime}=4$

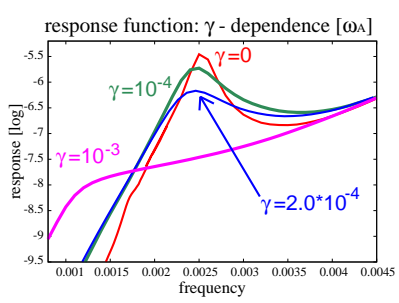

Figure 14. dependence of the response function on the growth rate $\gamma$

the overall damping, one has to fold the non-adiabatic response with the eigenfunction. This it not carried out here in detail, however it is obvious that the $q=4$ surface contributes most since although the non-adiabatic responses for $q=3$ and for $q=4$ are comparable, the eigenfunction is much larger at $\varrho_{p o l}=0.92(q=4)$.

As a last point the dependence of the response on a finite growth rate $\gamma$ is investigated. Typical growth rates for RWMs are very small $\left(\gamma \leq 10^{-3} \omega_{A}\right)$ - small enough to be stabilised by a set of external feedback coils. A finite $\gamma$ changes the resonance denominator in equations 7 and 8 and moves the pole(s) into the complex plane. The resonance becomes less sharp and therefore the width of the mode peak in the response function broadens (fig. 14). If $\gamma$ becomes comparable to the real mode frequency then there is no proper wave resonance any more and the peak completely disappears.

From the discussion above one can conclude that the RWM mode is mainly damped by ion acoustic damping. In addition, if the local ion diamagnetic drift frequency becomes comparable to the rotation frequency, electrostatic effects near the rational surfaces play a role and lead to modes that are less damped than the ones in the surrounding frequency regions.

The limitations of this model are obviously a) that the eigenfunction is not calculated self-consistently and therefore the radial shape differs slightly from the CASTOR eigenfunction, b) that there is no distinction between co- and counter-passing background particles in the propagator coefficients $\hat{a}_{k m}$ and $K_{m, m^{\prime}, k}$ what might broaden the peaks also slightly and c) that there are no collisions included. The last point seems to be the most severe limitation, but since the local ion collision frequency at the $q=4$ surface is about $0.0016 \omega_{A}$ or $1 \mathrm{kHz}$, the first and strongest response peak (mode1 in fig. 11 ) is expected to persist even when collisions are included. 


\section{Conclusions}

The first part of the paper presents the kinetic model and numerical benchmarks/results concerning low frequency modes in tokamak plasmas. Both local and global solutions for electrostatic (IAW), electromagnetic (KAW) and drift-type modes are shown. The possibility to switch easily between global and local for the same background parameters allows one to find both the complex dispersion relation and the global eigenfunction together with its damping or growth rate. Radial grid refinement allows for sub- $\varrho_{i^{-}}$ resolution and therefore for thorough numerical convergence checks.

The second part is an application of this multi-scale-length extension of LIGKA: the main kinetic damping mechanism of the external kink mode is sound wave damping, as also other publications have concluded before. However, for the first time also magnetic and diamagnetic drifts were included. It was found that there may exist less damped modes if the local ion diamagnetic drift frequency at a certain rational surface becomes comparable to the rotation frequency. A detailed quantitative analysis and comparisons with previously published results [20] lie beyond the scope of this paper and will be carried out in the next future.

\section{References}

[1] G.Y. Fu, J.W. VanDam, Phys. Fluids 1, 1949 (1989)

[2] R.R. Mett, S.M. Mahajan, Phys. Fluids B 4, 2885 (1992)

[3] C.Z. Cheng, L. Chen, M.S. Chance, Annals of Physics 161, 21 (1985)

[4] C.Z. Cheng, M.S. Chance, Phys. Fluids 29, 11 (1986)

[5] H. Kimura et al, Nucl. Fusion 38, 1303 (1998)

[6] D. Borba, W. Kerner, J. Comput. Phys 153, 101 (1999)

[7] H.L Berk, J.W. Van Dam, Z. Guo, and D.M. Lindberg, Phys. Fluids B 4, 1806 (1992)

[8] B.N. Breizman, S.E. Sharapov, Plasma Phys Control. Fusion 37, 1057 (1996)

[9] A. Jaun et al, Plasma Phys Control. Fusion 39, 549 (1997)

[10] P. Lauber et al, 'Damping rates of global kinetic modes in tokamaks', 3rd IAEA technical meeting on Theory of Plasma Instabilities, York, 26-28 March (2007)

[11] Ph. Lauber, 'Linear Gyrokinetic Description of Fast Particle Effects on the MHD Stability in Tokamaks', Ph.D. Thesis, TU München, (2003)

[12] Ph. Lauber, S. Günter, A, Könies, S.D. Pinches, J. Comp. Phys. 226, 447 (2007)

[13] C.Z. Cheng, Phys. Rep. 1, 211 (1992)

[14] H. Qin, 'Gyrokinetic Theory and Computational Methods for Electromagnetic Perturbations in Tokamaks', Ph.D. Thesis, Princeton University (1998)

[15] H. Qin,W.M. Tang, G.Rewoldt, Phys. Plasmas 5, 1035 (1998)

[16] S. D. Pinches, 'Nonlinear Interaction of Fast Particles with Alfvén Waves in Tokamaks', Ph.D. Thesis, The University of Nottingham (1996)

[17] S. D. Pinches, L.C. Appel, J. Candy et al., CPC 111, 131 (1998)

[18] A. Bondeson, D.J. Ward, Phys. Rev. Lett. 72, 2709 (1994)

[19] D. Gregoratto et al, Plasma Phys. Control. Fusion 43, 1425 (2001)

[20] Y. Liu et al, Nucl. Fusion 45, 1131 (2005)

[21] G.T.A. Huysmans et al., Phys. Fluids B5, 1545 (1993)

[22] H.L Berk, R.R. Mett, and D.M. Lindberg, Phys. Fluids B 5, 3969 (1993)

[23] G.Y. Fu, H. L. Berk, A. Pletzer, Phys. Plasmas 12, 082505 (2005) 
[24] G.T.A. Huysmans. J.P. Goedbloed, W. Kerner, Proc. CP90 Conf. on Comp Phys.Proc., World Scientific Publ.Co., 371 (1991)

[25] A. Fasoli et al , Phys. Rev. Lett. 75, 645 (1995) 